\title{
Distributed Generator and Load-Side Secondary Frequency Control in Power Networks
}

\author{
Changhong Zhao, Enrique Mallada, and Steven H. Low \\ Department of Computational and Mathematical Sciences \\ California Institute of Technology, Pasadena, California 91125 \\ Email: \{czhao, mallada, slow\}@ caltech.edu
}

\begin{abstract}
We design a distributed secondary frequency control scheme for both generators and controllable loads. The proposed scheme operates via local sensing and computation, and neighborhood communication. Equilibrium and stability analysis of the closed-loop system is performed with a power network model including turbines and governors of generators and nonlinear AC power flows. After a change in power supply or demand, the proposed scheme is able to stabilize the system, restore bus frequencies and net inter-area power exchanges, and minimize total generation cost minus user utility at equilibrium.
\end{abstract}

\section{INTRODUCTION}

It is important to control the frequency in power networks to keep it close to the nominal value (e.g., $60 \mathrm{~Hz}$ ). Frequency control is traditionally performed on the generator side, and it is composed of three layers: primary, secondary and economic dispatch, which operate in concert from fast to slow timescales [1]. Among them, the secondary frequency control operates on a timescale up to a minute or so and adjusts generators within each control area in a centralized manner, to restore area frequencies and net inter-area power exchanges to their nominal values. Recent work also designed distributed secondary frequency control schemes for bulk generators [2][4] or microgrids [5]. These schemes are scalable, flexible and economically efficient, and show great potential for future grids with a large number of distributed energy resources.

On the other hand, flexible and controllable loads may also participate in frequency control. This idea was first proposed by Schweppe et al. [6], and has been explored as a faster and cleaner supplement to the generator-side scheme, in order to reduce spinning reserves needed to balance intermittent and volatile renewable generations. Load-side frequency control has been studied in simulations and small-scale trials [7]-[9]. We have designed decentralized primary [10] and distributed secondary [11] load-side frequency control and analytically proved their stability under a linearized power network model. Recent work also initiated the study of joint generator and load-side distributed frequency control [12], [13].

In this paper, we propose a distributed secondary frequency control scheme that operates jointly on generators and loads. The proposed scheme is based on local sensing and computation, and communication between neighbors. After an unexpected change in power injection, the proposed scheme is able to stabilize the system to an equilibrium where bus frequencies and net inter-area power exchanges are restored to their nominal values, and the total generation cost minus user utility is minimized. Under mild conditions, we prove local asymptotic stability of the closed-loop system, with a relatively realistic power network model including governorturbine dynamics and nonlinear AC power flows. We demonstrate performance of the proposed scheme by simulations with a more realistic model in Power System Toolbox [14].

Instead of jointly designing generator and load-side control using the method in this paper, we can also design just the load-side control, and keep the traditional droop control on the generator side. The stability result in this paper says that if the generator-side control satisfies a certain condition, then the closed-loop system is stable. For this reason, we formulate the equilibrium objectives of our control as an optimal load control (OLC) problem, which uses the name in our previous papers [10], [11]. Here OLC is a class of problems for this kind of frequency control designs. It can be for primary and/or secondary frequency controls, under linear or nonlinear models, with or without design of generator-side control.

The rest of this paper is organized as follows. Section II introduces a dynamic power network model and formulates the OLC problem. Section III proposes the distributed secondary frequency control scheme. Sections IV and V perform equilibrium and stability analysis of the closed-loop system. Section VI shows the simulation results. Section VII concludes the paper and discusses future work.

\section{PROBlem Formulation}

Let $\mathbb{R}$ be the set of real numbers and $\mathbb{N}$ the set of natural numbers. Given a finite set $S \subset \mathbb{N}$ we use $|S|$ to denote its cardinality. For a set of scalar numbers $\left\{a_{i} \in \mathbb{R} \mid i \in S\right\}$, we use $a_{S}$ to denote the column vector of the $a_{i}$ 's; we usually drop the subscript $S$ when $S$ is clear from the context. For two vectors $a \in \mathbb{R}^{|S|}$ and $b \in \mathbb{R}^{\left|S^{\prime}\right|},(a, b) \in \mathbb{R}^{|S|+\left|S^{\prime}\right|}$ is a column vector. Given any matrix $A$, we denote its transpose by $A^{T}$, and its $i$-th row by $A_{i}$. We use $A_{S}$ to denote the submatrix of $A$ composed only of the rows $A_{i}$ for $i \in S$. The diagonal matrix of a sequence $\left\{a_{i}, i \in S\right\}$ is represented by $\operatorname{diag}\left(a_{S}\right)=\operatorname{diag}\left(a_{i}, i \in S\right)$, or $a_{S}$ for short when its meaning is clear. Finally, we use 1(0) to denote the vector/matrix of all ones (zeros), whose dimension is understood from the context.

\section{A. Dynamic Power Network Model}

We consider a classical power network model [1] described by a directed graph $(\mathcal{N}, \mathcal{E})$, where $\mathcal{N}:=\{1, \ldots,|\mathcal{N}|\}$ is the set of buses (nodes) and $\mathcal{E} \subset \mathcal{N} \times \mathcal{N}$ is the set of transmission lines. A line is denoted interchangeably by $e \in \mathcal{E}$, or $i j \in$ $\mathcal{E}$ if it is directed from buses $i$ to $j$. We partition the buses 
$\mathcal{N}=\mathcal{G} \cup \mathcal{L}$ where $\mathcal{G}$ and $\mathcal{L}$ are the set of generator and load buses respectively. We assume $(\mathcal{N}, \mathcal{E})$ is connected, bus voltage magnitudes $\left|V_{i}\right|=1$ pu for all $i \in \mathcal{N}$, and all lines $i j \in \mathcal{E}$ are lossless with susceptances $B_{i j}>0$.

We introduce the following parameters and variables:

- $\quad$ Let bus voltage phase angles be $\theta:=\left(\theta_{i}, i \in \mathcal{N}\right)$ and bus frequency deviations from the nominal value be $\omega:=\left(\omega_{i}, i \in \mathcal{N}\right)$. Define $\tilde{\theta}:=T \theta$ where

$$
T:=\left[I_{(|\mathcal{N}|-1) \times(|\mathcal{N}|-1)},-1_{|\mathcal{N}|-1}\right]
$$

and $1_{|\mathcal{N}|-1}$ is an $(|\mathcal{N}|-1)$-dimensional vector with each entry being " 1 ", such that $\tilde{\theta}_{i}=\theta_{i}-\theta_{|\mathcal{N}|}$ for $i=1, \ldots,|\mathcal{N}|-1$.

- Denote uncontrollable bus power injections by $r:=$ $\left(r_{i}, i \in \mathcal{N}\right)$, which is a constant such that it models the power supply or demand after an unexpected step change. Let $p_{\mathcal{G}}^{M}:=\left(p_{i}^{M}, i \in \mathcal{G}\right)$ be the mechanic power injections of generators, and $d:=\left(d_{i}, i \in \mathcal{N}\right)$ be the power consumptions of controllable loads. ${ }^{1}$

- The aggregate power of generator damping and frequency-dependent (uncontrollable) loads at bus $i \in$ $\mathcal{N}$ is $D_{i} \omega_{i}$, with $D_{i}>0$ a constant. The inertia constant of generator $i \in \mathcal{G}$ is $M_{i}$. Define $D_{\mathcal{G}}:=$ $\operatorname{diag}\left(D_{i}, \quad i \in \mathcal{G}\right), D_{\mathcal{L}}=\operatorname{diag}\left(D_{i}, \quad i \in \mathcal{L}\right)$, and $M_{\mathcal{G}}:=\operatorname{diag}\left(M_{i}, i \in \mathcal{G}\right)$.

- The incidence matrix $C \in \mathbb{R}^{|\mathcal{N}| \times|\mathcal{E}|}$ is defined such that its $(j, e)$-th component $C_{j, e}=1$ if line $e=j k$ is directed from bus $j$ to some bus $k, C_{j, e}=-1$ if $e=i j$ is directed from some bus $i$ to bus $j$, and $C_{j, e}=0$ otherwise. Let $\tilde{C}$ be the reduced incidence matrix by removing the last row of $C$. Let line susceptances be arranged in a diagonal matrix $B:=\operatorname{diag}\left(B_{i j}, i j \in \mathcal{E}\right)$, and line power flows be $p:=\left(p_{i j}, i j \in \mathcal{E}\right)$.

Then we have

$$
\begin{aligned}
\dot{\tilde{\theta}} & =T \omega \\
M_{\mathcal{G}} \dot{\omega}_{\mathcal{G}} & =r_{\mathcal{G}}+p_{\mathcal{G}}^{M}-D_{\mathcal{G}} \omega_{\mathcal{G}}-d_{\mathcal{G}}-C_{\mathcal{G}} p \\
0 & =r_{\mathcal{L}}-D_{\mathcal{L}} \omega_{\mathcal{L}}-d_{\mathcal{L}}-C_{\mathcal{L}} p \\
p & =B \sin \left(\tilde{C}^{T} \tilde{\theta}\right)
\end{aligned}
$$

where (2) is the swing equations of generators, (3) is the power balance equations on load buses, and (4) is nonlinear AC power flow equations. Moreover, we consider a classical governorturbine model in generators [1]:

$$
\begin{aligned}
\tau_{g, i} \dot{a}_{i} & =-a_{i}+p_{i}^{C} & & i \in \mathcal{G} \\
\tau_{b, i} \dot{p}_{i}^{M} & =-p_{i}^{M}+a_{i} & & i \in \mathcal{G}
\end{aligned}
$$

where $p_{i}^{C}$ is the generator control command, $a_{i}$ is the position of valve, and time constants $\tau_{g, i}$ and $\tau_{b, i}$ characterize time-delay in governor response and fluid dynamics in the turbine respectively. By using (5)(6) we ignore the traditional droop control, since a redesign of the generator-side frequency control will be proposed below. As we will see, the redesign includes the traditional droop control as a special case.

\footnotetext{
${ }^{1}$ We assume one controllable load on every bus, while our results can be generalized to cases with multiple or no controllable loads on some buses.
}

The power network dynamics are hence described by (1)$(6)$, where $\left(p_{\mathcal{G}}^{C}, d\right)$ are control variables and $\left(\tilde{\theta}, \omega, p, p_{\mathcal{G}}^{M}, a_{\mathcal{G}}\right)$ are state variables.

\section{B. Operational Constraints}

We introduce some operational constraints which will be considered in the problem formulated in Section II-C.

First, the power network is partitioned into several control areas. Let $\mathcal{K}$ be the set of control areas, and define matrix $E \in\{0,1\}^{|\mathcal{K}| \times|\mathcal{N}|}$ such that $E_{k, i}=1$ if bus $i$ lies in area $k$, and $E_{k, i}=0$ otherwise. We impose the constraint

$$
E C p=\hat{p}
$$

which says that the net inter-area power exchanges must be equal to their nominal values $\hat{p}:=\left(\hat{p}_{k}, k \in \mathcal{K}\right)$.

Remark 1: The constraint (7) can be generalized in the following way. For any given subset $\mathcal{M} \subseteq \mathcal{N}$ of buses, define a row vector $E_{\mathcal{M}} \in\{0,1\}^{1 \times|\mathcal{N}|}$ such that its $i$-th component $E_{\mathcal{M}, i}=1$ if bus $i \in \mathcal{M}$, and $E_{\mathcal{M}, i}=0$ otherwise. Then the inequality constraint

$$
\underline{p}_{\mathcal{M}} \leq E_{\mathcal{M}} C p \leq \bar{p}_{\mathcal{M}}
$$

limits the net power exchange out of $\mathcal{M}$ within $\left[\underline{p}_{\mathcal{M}}, \bar{p}_{\mathcal{M}}\right]$. If the power flow $p_{i j}$ on a line $i j \in \mathcal{E}$ can be expressed as $p_{i j}=E_{\mathcal{M}} C p$ for some $\mathcal{M}$, then (8) indeed imposes thermal constraint $\underline{p}_{\mathcal{M}} \leq p_{i j} \leq \bar{p}_{\mathcal{M}}$. This holds if and only if line $i j$ itself forms a cutset of the graph. Some lines may not satisfy this condition in power transmission networks which have a mesh topology. It is our future work to explore how to impose thermal constraints on all lines.

Second, we define

$$
\tilde{\Theta}:=\left\{\tilde{\theta} \in \mathbb{R}^{|\mathcal{N}|-1}|| \tilde{\theta}_{i}-\tilde{\theta}_{j} \mid<\pi / 2, \forall i j \in \mathcal{E}\right\}
$$

where $\tilde{\theta}_{|\mathcal{N}|} \equiv 0$ is defined for convenience. In practice, phase angles should always fall in $\tilde{\Theta}$, as a security requirement [1].

Finally, let the operating regions of generators be $p_{i}^{C} \in$ $\mathcal{P}_{i}:=\left[p_{i}, \bar{p}_{i}\right]$ and those of controllable loads be $d_{i} \in \mathcal{D}_{i}:=$ $\left[\underline{d}_{i}, \bar{d}_{i}\right]$, and $\mathcal{P}_{\mathcal{G}}:=\Pi_{i \in \mathcal{G}} \mathcal{P}_{i}$ and $\mathcal{D}:=\Pi_{i \in \mathcal{N}} \mathcal{D}_{i}$. The region $\mathcal{B}$ of power flows $p$ is defined as $\mathcal{B}:=\Pi_{e \in \mathcal{E}} \mathcal{B}_{e}$ where $\mathcal{B}_{e}:=$ $\left[-B_{e}, B_{e}\right]$, since on a line $e=i j$, the power flow $p_{e}=$ $B_{e} \sin \left(\tilde{\theta}_{i}-\tilde{\theta}_{j}\right)$.

\section{Optimal Load Control Problem}

Given uncontrollable power injections $r \in \mathbb{R}^{|\mathcal{N}|}$, our goal is to design controllers for $\left(p_{\mathcal{G}}^{C}, d\right)$ to stabilize the system (1)(6) at an equilibrium $\left(p_{\mathcal{G}}^{C, *}, d^{*} ; \tilde{\theta}^{*}, \omega^{*}, p^{*}, p_{\mathcal{G}}^{M, *}, a_{\mathcal{G}}^{*}\right)$ where

(i) (Frequency restoration) $\omega^{*}=0$;

(ii) (Phase angle security) $\tilde{\theta}^{*} \in \tilde{\Theta}$;

(iii) (Economic efficiency) $\left(p_{\mathcal{G}}^{C, *}, d^{*}, p^{*}\right)$ solves the optimal load control (OLC) problem.

OLC:

$$
\begin{aligned}
\min _{\substack{p_{\mathcal{G}}^{C}, d, p \\
\text { subject to }}} & \sum_{i \in \mathcal{G}} G_{i}\left(p_{i}^{C}\right)-\sum_{i \in \mathcal{N}} U_{i}\left(d_{i}\right) \\
& E C \hat{p}
\end{aligned}
$$




$$
\begin{aligned}
& r+p^{C}-d-C p=0^{2} \\
& p_{\mathcal{G}}^{C} \in \mathcal{P}_{\mathcal{G}}, \quad d \in \mathcal{D}, \quad p \in \mathcal{B} .
\end{aligned}
$$

The objective (9) of OLC is to minimize the total generation cost $G_{i}$ for all $i \in \mathcal{G}$ minus total user utility $U_{i}$ resulting from controllable loads $d_{i}$ for all $i \in \mathcal{N}$, subject to net inter-area power exchange constraint (10), bus power balance (11), and operating regions (12).

We define $G_{i}$ on the open domain $\mathcal{P}_{i}^{\epsilon}:=\left(\underline{p}_{i}-\epsilon, \bar{p}_{i}+\epsilon\right)$, and $U_{i}$ on $\mathcal{D}_{i}^{\epsilon}:=\left(\underline{d}_{i}-\epsilon, \bar{d}_{i}+\epsilon\right)$, for some $\epsilon>0$. We make the following assumptions:

Assumption 1: For all $i \in \mathcal{G}(i \in \mathcal{N}), G_{i}\left(U_{i}\right)$ is secondorder continuously differentiable with $G_{i}^{\prime \prime}(\cdot) \geq 1 / \bar{\alpha}_{i}>0$ in $\mathcal{P}_{i}^{\epsilon}\left(U_{i}^{\prime \prime}(\cdot) \leq-1 / \bar{\beta}_{i}<0\right.$ in $\left.\mathcal{D}_{i}^{\epsilon}\right)$.

Assumption 2: OLC is feasible.

Let $\pi \in \mathbb{R}^{|\mathcal{K}|}, \lambda \in \mathbb{R}^{|\mathcal{N}|}$, and $\rho \in \mathbb{R}^{2(|\mathcal{G}|+|\mathcal{N}|+|\mathcal{E}|)}$ be Lagrange multipliers of constraints (10), (11), and (12) respectively. We have:

Proposition 1: Suppose Assumptions 1 and 2 hold. Then there exist $\left(p_{\mathcal{G}}^{C, *}, d^{*}, p^{*}\right)$ and $\left(\pi^{*}, \lambda^{*}, \rho^{*}\right)$ which are optimal for OLC and its dual, with zero duality gap. Moreover, $\left(p_{\mathcal{G}}^{C, *}, d^{*}\right)$ is unique for all the optimal solutions of OLC.

Proof: By Assumptions 1 and 2, OLC is a convex problem whose feasible set is nonempty and compact. Hence an optimal $\left(p_{\mathcal{G}}^{C, *}, d^{*}, p^{*}\right)$ exists. Moreover, $\left(p_{\mathcal{G}}^{C, *}, d^{*}\right)$ is unique for all the optimal solutions of OLC since the objective function (9) is strictly convex in $\left(p_{\mathcal{G}}^{C}, d\right)$.

Since all the constraints of OLC are affine, Assumption 2 implies zero duality gap. Clearly, the primal and dual optimal objective values are equal and finite, and hence a dual optimal solution exists [15, Ch. 5.2.3].

Proposition 1, however, guarantees neither the uniqueness of optimal $p^{*}$ of OLC, nor the existence of $\tilde{\theta}^{*}$ which solves power flow equations $p^{*}=B \sin \left(\tilde{C}^{T} \tilde{\theta}^{*}\right)$ for any optimal $p^{*}$. Define the image set of power flow equations

$$
p(\tilde{\Theta}):=\left\{p \in \mathbb{R}^{|\mathcal{E}|} \mid p=B \sin \left(\tilde{C}^{T} \tilde{\theta}\right) \text { for some } \tilde{\theta} \in \tilde{\Theta}\right\} .
$$

We make the following assumption.

Assumption 3: There is at least one optimal solution $\left(p_{\mathcal{G}}^{C, *}, d^{*}, p^{*}\right)$ of OLC such that $p^{*} \in p(\tilde{\Theta})$.

Assumption 3 assumes existence of an optimal OLC solution which ensures feasibility and phase angle security of power flow equations. It implies uniqueness of such an optimal solution, as shown by the following proposition.

Proposition 2: Suppose Assumptions 1, 2 and 3 hold. Then there is a unique $\left(p_{\mathcal{G}}^{C, *}, d^{*}, p^{*}, \tilde{\theta}^{*}\right)$ such that $\left(p_{\mathcal{G}}^{C, *}, d^{*}, p^{*}\right)$ is optimal for OLC, $\tilde{\theta}^{*} \in \tilde{\Theta}$, and $p^{*}=B \sin \left(\tilde{C}^{T} \tilde{\theta}^{*}\right)$.

Proof: Since Assumptions 1 and 2 hold, Proposition 1 ensures the existence of optimal point(s) $\left(p_{\mathcal{G}}^{C, *}, d^{*}, p^{*}\right)$ of OLC and uniqueness of $\left(p_{\mathcal{G}}^{C, *}, d^{*}\right)$. By Assumption 3, there is an optimal $\left(p_{\mathcal{G}}^{C, *}, d^{*}, p^{*, 0}\right)$ such that $p^{*, 0}=B \sin \left(\tilde{C}^{T} \tilde{\theta}^{*, 0}\right)$

\footnotetext{
${ }^{2}$ We define constants $p_{\mathcal{L}}^{C}=p_{\mathcal{L}}^{M}=a_{\mathcal{L}}=p_{\mathcal{L}}^{C, *}=p_{\mathcal{L}}^{M, *}=a_{\mathcal{L}}^{*} \equiv 0$, and use $p^{C}:=\left(p_{\mathcal{G}}^{C}, p_{\mathcal{L}}^{C}\right) \in \mathbb{R}^{\lceil\mathcal{N} \mid}$ in (11) and similar notations below.
}

for some $\tilde{\theta}^{*, 0} \in \tilde{\Theta}$. Suppose there is another optimal point $\left(p_{\mathcal{G}}^{C, *}, d^{*}, p^{*, 1}\right)$ where $p^{*, 1} \neq p^{*, 0}$, such that $p^{*, 1}=$ $B \sin \left(\tilde{C}^{T} \tilde{\theta}^{*, 1}\right)$ for some $\tilde{\theta}^{*, 1} \in \tilde{\Theta}$. We have

$$
C B \sin \left(\tilde{C}^{T} \tilde{\theta}^{*, j}\right)=r+p^{C, *}-d^{*}, \quad \forall j=0,1
$$

due to constraint (11). Hence we must have $\theta^{*, 0}=\theta^{*, 1}$, since $C B \sin \left(\tilde{C}^{T}\right.$. $)$ is a one-to-one function on $\tilde{\Theta}$ [16]. Therefore $p^{*, 1}=p^{*, 0}$ and we get a contradiction. This implies uniqueness of optimal $\left(p_{\mathcal{G}}^{C, *}, d^{*}, p^{*}\right)$ with $p^{*} \in p(\tilde{\Theta})$, and also uniqueness of $\tilde{\theta}^{*} \in \tilde{\Theta}$ such that $p^{*}=B \sin \left(\tilde{C}^{T} \tilde{\theta}^{*}\right)$.

\section{Distributed SECONDARy FREQUENCy CONTROL}

Inspired by the approach in [11], we design the following distributed control scheme for $\left(p_{\mathcal{G}}^{C}, d\right)$ :

$$
\begin{aligned}
p_{\mathcal{G}}^{C} & =p_{\mathcal{G}}^{C}\left(\lambda_{\mathcal{G}}+\omega_{\mathcal{G}}\right), \quad d=d(\lambda+\omega) \\
\dot{\lambda} & =K^{\lambda}\left(M \dot{\omega}+D \omega+C p-C B \sin \left(C^{T} \phi\right)\right) \\
\dot{\pi} & =K^{\pi}\left(E C B \sin \left(C^{T} \phi\right)-\hat{p}\right) \\
\dot{\phi} & =\lambda-E^{T} \pi
\end{aligned}
$$

where $K^{\lambda}, K^{\pi}$ are diagonal matrices of positive gains, $M$ is the diagonal matrix with diagonal terms $\left(M_{i}, i \in \mathcal{G}\right)$ and $0_{\mathcal{L}}$. The $i$-th components of functions $p_{\mathcal{G}}^{C}(\cdot)$ for $i \in \mathcal{G}$ and $d(\cdot)$ for $i \in \mathcal{N}$ in (14) are defined as

$$
\begin{array}{r}
p_{i}^{C}(x):= \begin{cases}\bar{p}_{i} & \text { if } x<-G_{i}^{\prime}\left(\bar{p}_{i}\right) \\
\underline{p}_{i} & \text { if } x>-G_{i}^{\prime}\left(\underline{p}_{i}\right) \\
\left(G_{i}^{\prime}\right)^{-1}(-x) & \text { otherwise }\end{cases} \\
d_{i}(x):= \begin{cases}\underline{d}_{i} & \text { if } x<-U_{i}^{\prime}\left(\underline{d}_{i}\right) \\
\bar{d}_{i} & \text { if } x>-U_{i}^{\prime}\left(\bar{d}_{i}\right) \\
\left(U_{i}^{\prime}\right)^{-1}(-x) & \text { otherwise. }\end{cases}
\end{array}
$$

Assumption 1 guarantees inverse functions of $G_{i}^{\prime}$ and $U_{i}^{\prime}$ are well defined on $\left[G_{i}^{\prime}\left(\underline{p}_{i}\right), G_{i}^{\prime}\left(\bar{p}_{i}\right)\right]$ and $\left[U_{i}^{\prime}\left(\bar{d}_{i}\right), U_{i}^{\prime}\left(\underline{d}_{i}\right)\right]$, and functions $p_{\mathcal{G}}^{C}(\cdot)$ and $d(\cdot)$ are Lipschitz on $\mathbb{R}$ such that the system described by (1)-(6) and (14)-(17) has a unique trajectory starting from a given initial point.

The control scheme (14)-(17) computes auxiliary variables $\lambda, \phi \in \mathbb{R}^{|\mathcal{N}|}$ and $\pi \in \mathbb{R}^{|\mathcal{K}|}$ in real time. We use the same notations $\lambda, \pi$ for the auxiliary variables and the dual variables of OLC, for the reason which will become clear in Section IV. The proposed scheme is distributed in that it operates via local sensing and computation, and communication between neighbors. In (15), each bus $i \in \mathcal{N}$ computes $\lambda_{i}$ by locally sensing $\omega_{i}$ and the total power flow out of $i$, and receiving $\phi_{j}$ from its neighbors $j$. In (16), each area $k \in \mathcal{K}$ computes $\pi_{k}$ by monitoring the differences of $\phi$ across lines connecting area $k$ with other areas. It then broadcasts $\pi_{k}$ to all the buses in area $k$. In (17), each bus $i \in \mathcal{N}$ computes $\phi_{i}$ from its local $\lambda_{i}$ and the $\pi_{k}$ it receives. The centralized computation and broadcast of $\pi_{k}$ within each area $k$ are only needed when we impose net inter-area power exchange constraints.

For simplicity of the following study, we define $\tilde{\phi}:=T \phi$ where $T$ is defined in Section II-A, such that $\tilde{\phi}_{i}=\phi_{i}-\phi_{|\mathcal{N}|}$ for $i=1, \ldots,|\mathcal{N}|-1$. We also define a constant $\tilde{\phi}_{|\mathcal{N}|} \equiv 0$. By (2)(3), equations (14)-(17) can be rewritten as

$$
p_{\mathcal{G}}^{C}=p_{\mathcal{G}}^{C}\left(\lambda_{\mathcal{G}}+\omega_{\mathcal{G}}\right), \quad d=d(\lambda+\omega)
$$




$$
\begin{aligned}
& \dot{\lambda}=K^{\lambda}\left(r+p^{M}-d-C B \sin \left(\tilde{C}^{T} \tilde{\phi}\right)\right) \\
& \dot{\pi}=K^{\pi}\left(E C B \sin \left(\tilde{C}^{T} \tilde{\phi}\right)-\hat{p}\right) \\
& \dot{\tilde{\phi}}=T\left(\lambda-E^{T} \pi\right) .
\end{aligned}
$$

The system described by (1)-(6) and (18)-(21) is referred to as the closed-loop system.

\section{EQUILIBRIUM ANALYSIS}

We first show the following lemma on equilibrium condition of the closed-loop system.

\section{Lemma 1 (Equilibrium Condition):}

A point $\left(p_{\mathcal{G}}^{C, *}, d^{*} ; \tilde{\theta}^{*}, \omega^{*}, p^{*}, p_{\mathcal{G}}^{M, *}, a_{\mathcal{G}}^{*} ; \lambda^{*}, \pi^{*}, \tilde{\phi}^{*}\right)$ is an equilibrium of the closed-loop system if and only if

$$
\begin{aligned}
\omega^{*} & =0 \\
p_{\mathcal{G}}^{M, *} & =a_{\mathcal{G}}^{*}=p_{\mathcal{G}}^{C, *} \\
p^{*} & =B \sin \left(\tilde{C}^{T} \tilde{\theta}^{*}\right) \\
C p^{*} & =C B \sin \left(\tilde{C}^{T} \tilde{\phi}^{*}\right) \\
0 & =r+p^{C, *}-d^{*}-C p^{*} \\
\hat{p} & =E C p^{*} \\
p_{\mathcal{G}}^{C, *} & =p_{\mathcal{G}}^{C}\left(\lambda_{\mathcal{G}}^{*}\right), \quad d^{*}=d\left(\lambda^{*}\right) \\
\lambda^{*} & -E^{T} \pi^{*} \in \operatorname{span}\{1\} .
\end{aligned}
$$

Proof: Proof is skipped due to space limitation.

By the equilibrium condition in Lemma 1 and the results in Section II-C, we have the following theorem.

Theorem 1 (Properties of Closed-Loop Equilibria): Suppose Assumptions 1, 2 and 3 hold. Define

$$
E^{*}:=\left\{\text { equilibria of (1)-(6) and (18)-(21) } \mid \tilde{\theta}^{*} \in \tilde{\Theta}\right\} .
$$

Then $E^{*}$ is nonempty, and all the equilibria in $E^{*}$ have the same $\left(p_{\mathcal{G}}^{C, *}, d^{*} ; \theta^{*}, \omega^{*}, p^{*}, p_{\mathcal{G}}^{M, *}, a_{\mathcal{G}}^{*}\right)$, where $\omega^{*}=0$ and $\left(p_{\mathcal{G}}^{C, *}, d^{*}, p^{*}\right)$ is an optimal solution of OLC.

Proof: By Propositions 1 and 2, there are $\left(p_{\mathcal{G}}^{C, *}, d^{*}, p^{*}, \tilde{\theta}^{*}\right)$ and $\left(\pi^{*}, \lambda^{*}, \rho^{*}\right)$ such that

(i) $\quad \tilde{\theta}^{*} \in \tilde{\Theta}$ and $p^{*}=B \sin \left(\tilde{C}^{T} \tilde{\theta}^{*}\right)$;

(ii) $\left(p_{\mathcal{G}}^{C, *}, d^{*}, p^{*}\right)$ and $\left(\pi^{*}, \lambda^{*}, \rho^{*}\right)$ are optimal for OLC and its dual.

Hence $\left(p_{\mathcal{G}}^{C, *}, d^{*}, p^{*}\right)$ and $\left(\pi^{*}, \lambda^{*}, \rho^{*}\right)$ satisfy the Karush-KuhnTucker (KKT) conditions [15, Ch. 5.5.3], which are equivalently written as

$$
\begin{aligned}
& r+p^{C, *}-d^{*}-C p^{*}=0 \\
& E C p^{*}=\hat{p} \\
& p_{\mathcal{G}}^{C, *}=p_{\mathcal{G}}^{C}\left(\lambda_{\mathcal{G}}^{*}\right), \quad d^{*}=d\left(\lambda^{*}\right) \\
& C^{T} E^{T} \pi^{*}-C^{T} \lambda^{*}=0
\end{aligned}
$$

where (30)(31) are primal feasibility, and (32)(33) combine stationarity, dual feasibility, and complementary slackness. Indeed the components of $\rho^{*}$ associated with constraints $p_{\mathcal{G}}^{C} \in \mathcal{P}_{\mathcal{G}}$, $d \in \mathcal{D}$ are eliminated due to the projections in $p_{\mathcal{G}}^{C}(\cdot)$ and $d(\cdot)$, and the components associated with $p \in \mathcal{B}$ are zero since $p^{*} \in p(\tilde{\Theta})$ lies in the interior of $\mathcal{B}$. Note that (30)-(33) are equivalent to (26)-(29). Taking $\omega^{*}=0, p_{\mathcal{G}}^{M, *}=a_{\mathcal{G}}^{*}=p_{\mathcal{G}}^{C, *}$, and $\tilde{\phi}^{*}=\tilde{\theta}^{*}$, we get a point which satisfies the equilibrium condition (22)-(29) and $\tilde{\theta}^{*} \in \tilde{\Theta}$, i.e., is in $E^{*}$.

For all the equilibria which satisfy $\tilde{\theta}^{*} \in \tilde{\Theta}$, equations (26)(29) and hence the KKT conditions are satisfied. Therefore the components $\left(p_{\mathcal{G}}^{C, *}, d^{*}, p^{*}\right)$ and $\left(\pi^{*}, \lambda^{*}\right)$ of these equilibria are optimal for OLC and its dual. By Proposition 2, $\left(p_{\mathcal{G}}^{C, *}, d^{*}, p^{*}, \tilde{\theta}^{*}\right)$ is unique for all such equilibria. We have $\omega^{*}=0$ by (22) and uniqueness of $\left(p_{\mathcal{G}}^{M, *}, a_{\mathcal{G}}^{*}\right)$ by (23).

Theorem 1 has two implications. First, if we only consider physical variables such as generation, load, power flows, bus frequencies, etc. (as opposed to auxiliary variables $\lambda, \pi, \phi$ ), and only consider equilibria which ensure phase angle security, then the closed-loop system has a unique equilibrium. Second, this equilibrium satisfies all the requirements for secondary frequency control proposed at the beginning of Section II-C, in particular power balance, restoration of frequency and interarea power exchanges, and economic efficiency.

In Section V, we will study the stability of equilibria in a subset of $E^{*}$, which is defined in the following corollary.

Corollary 1: Suppose Assumptions 1, 2 and 3, and hence Theorem 1 hold. Define

$$
E_{\Phi}^{*}:=\left\{\text { equilibria in } E^{*} \mid \tilde{\phi}^{*} \in \tilde{\Theta}\right\} .
$$

Then $\underset{\sim}{E_{\Phi}^{*}}$ is nonempty, and all the equilibria in $E_{\Phi}^{*}$ have the same $\tilde{\phi}^{*}=\tilde{\theta}^{*}$.

Proof: Use Theorem 1, equation (25) and the fact that $C B \sin \left(\tilde{C}^{T} \cdot\right)$ is one-to-one on $\tilde{\Theta}$.

\section{Stability Analysis}

We use Lyapunov method to study the stability of the closed-loop equilibria in $E_{\Phi}^{*}$. Fix any equilibrium $\left(p_{\mathcal{G}}^{C, *}, d^{*} ; \tilde{\theta}^{*}, \omega^{*}, p^{*}, p_{\mathcal{G}}^{M, *}, a_{\mathcal{G}}^{*} ; \lambda^{*}, \phi^{*}, \pi^{*}\right) \in E_{\Phi}^{*}$. Define $\Delta \lambda:=\lambda-\lambda^{*}$ and use similar notations for deviations of other variables from this equilibrium. Define $\tilde{\theta}_{i j}:=\tilde{\theta}_{i}-\tilde{\theta}_{j}$ and $\tilde{\phi}_{i j}:=\tilde{\phi}_{i}-\tilde{\phi}_{j}$ for $i j \in \mathcal{E}$. Following [12], we choose the following Lyapunov function candidate, which is centered at the particular equilibrium we have chosen.

$$
V=V_{0}+\sum_{i \in \mathcal{G}} V_{i}
$$

where

$$
\begin{aligned}
V_{0}= & \frac{1}{2} \Delta \omega_{\mathcal{G}}^{T} M_{\mathcal{G}} \Delta \omega_{\mathcal{G}}+\sum_{i j \in \mathcal{E}} B_{i j} \int_{\tilde{\theta}_{i j}^{*}}^{\tilde{\theta}_{i j}}\left(\sin u-\sin \tilde{\theta}_{i j}^{*}\right) d u \\
& +\sum_{i j \in \mathcal{E}} B_{i j} \int_{\tilde{\phi}_{i j}^{*}}^{\tilde{\phi}_{i j}}\left(\sin u-\sin \tilde{\phi}_{i j}^{*}\right) d u \\
& +\frac{1}{2} \Delta \lambda^{T}\left(K^{\lambda}\right)^{-1} \Delta \lambda+\frac{1}{2} \Delta \pi^{T}\left(K^{\pi}\right)^{-1} \Delta \pi
\end{aligned}
$$

and

$$
V_{i}=\frac{1}{2}\left[\Delta a_{i}, \Delta p_{i}^{M}\right] P_{i}\left[\Delta a_{i}, \Delta p_{i}^{M}\right]^{T} \quad i \in \mathcal{G}
$$

for some positive definite matrices $P_{i} \in \mathbb{R}^{2 \times 2}$. 
By proving $V$ in (34) is a Lyapunov function under certain conditions, we have the following theorem.

Theorem 2 (Stability of Equilibria):

Suppose Assumptions 1, 2 and 3, and hence Theorem 1 and Corollary 1 hold. Suppose all of the following hold for all the equilibria in $E_{\Phi}^{*}$ (which have the same $d^{*}$ ):

(i) $\quad d^{*} \in \operatorname{Interior}(\mathcal{D})$, which implies uniqueness of $\lambda^{*}$;

(ii) $\quad U_{i}^{\prime \prime}(\cdot) \geq-1 / \underline{\beta}_{i}$ in a neighborhood of $d_{i}^{*}$ for $i \in \mathcal{N}$;

(iii) There exist $L_{i}^{-}$such that $0 \leq L_{i}<\underline{\beta}_{i}$ and $\mid p_{i}^{C}\left(x_{i}\right)-$ $p_{i}^{C}\left(\lambda_{i}^{*}\right)\left|\leq L_{i}\right| x_{i}-\lambda_{i}^{*} \mid$ for all $x_{i}$ in a neighborhood of $\lambda_{i}^{*}$, for all $i \in \mathcal{G}$.

Then any trajectory starting in a neighborhood of $E_{\Phi}^{*}$ converges to an equilibrium in $E_{\Phi}^{*}$.

Proof: Condition (i) implies uniqueness of $\lambda^{*}$ across $E_{\Phi}^{*}$ (indeed $E^{*}$ ) since $d^{*}$ is unique and functions $d_{i}(\cdot)$ are strictly increasing on $\left(-U_{i}^{\prime}\left(\underline{d}_{i}\right),-U_{i}^{\prime}\left(\bar{d}_{i}\right)\right)$ for all $i \in \mathcal{N}$. This does not imply $E_{\Phi}^{*}$ is a singleton, due to the nonuniqueness of $\pi^{*}$. By (29), if $\left(x^{*}, \pi^{*}\right) \in E_{\Phi}^{*}$ where $x$ denotes all the components except $\pi$, then $\left(x^{*}, \pi^{*}+1 a\right) \in E_{\Phi}^{*}$, for any scalar number $a$.

Take any equilibrium in $E_{\Phi}^{*}$ as the center of Lyapunov function candidate $V$ in (34)-(36). In a neighborhood of the equilibrium, $V$ is positive definite, and is zero only at the equilibrium [12]. Moreover, the time derivative of $V_{0}$ along any trajectory of the system is

$$
\begin{aligned}
\dot{V}_{0}= & \omega_{\mathcal{G}}^{T}\left\{r_{\mathcal{G}}+p_{\mathcal{G}}^{M}-D_{\mathcal{G}} \omega_{\mathcal{G}}-d_{\mathcal{G}}-C_{\mathcal{G}} B \sin \left(\tilde{C}^{T} \tilde{\theta}\right)\right\} \\
& +\omega^{T}\left\{C B \sin \left(\tilde{C}^{T} \tilde{\theta}\right)-C B \sin \left(\tilde{C}^{T} \tilde{\theta}^{*}\right)\right\} \\
& +\left(\Delta \lambda-E^{T} \Delta \pi\right)^{T}\left\{C B \sin \left(\tilde{C}^{T} \tilde{\phi}\right)-C B \sin \left(\tilde{C}^{T} \tilde{\phi}^{*}\right)\right\} \\
& +\Delta \lambda^{T}\left\{r+p^{M}-d-C B \sin \left(\tilde{C}^{T} \tilde{\phi}\right)\right\} \\
& +\Delta \pi^{T}\left\{E C B \sin \left(\tilde{C}^{T} \tilde{\phi}\right)-\hat{p}\right\} \\
= & \omega^{T}\left\{r+p^{M}-D \omega-d-C B \sin \left(\tilde{C}^{T} \tilde{\theta}^{*}\right)\right\} \\
& +\Delta \lambda^{T}\left\{r+p^{M}-d-C B \sin \left(\tilde{C}^{T} \tilde{\phi}^{*}\right)\right\} \\
& +\Delta \pi^{T}\left\{E C B \sin \left(\tilde{C}^{T} \tilde{\phi}^{*}\right)-\hat{p}\right\} \\
= & -\Delta \omega^{T} D \Delta \omega-(\Delta \omega+\Delta \lambda)^{T} \Delta d \\
& +\left(\Delta \omega_{\mathcal{G}}+\Delta \lambda_{\mathcal{G}}\right)^{T} \Delta p_{\mathcal{G}}^{M}
\end{aligned}
$$

where we use $\omega$ and $\Delta \omega$ interchangeably since $\omega^{*}=0$. We get (37) by system dynamics (1)(2)(4) and (19)-(21) and the fact that $\lambda^{*}-E^{T} \pi^{*} \in \operatorname{span}\{1\}$, get (38) by (3)(4), and get (39) by equilibrium conditions $C B \sin \left(\tilde{C}^{T} \tilde{\theta}^{*}\right)=r+p^{M, *}-d^{*}$ and (23), (25)-(27). By conditions (i)(ii) we have

$$
\begin{aligned}
& -(\Delta \omega+\Delta \lambda)^{T} \Delta d \\
= & -\sum_{i \in \mathcal{N}}\left(\Delta \omega_{i}+\Delta \lambda_{i}\right)\left(d_{i}\left(\omega_{i}+\lambda_{i}\right)-d_{i}\left(\lambda_{i}^{*}\right)\right) \\
\leq & -\sum_{i \in \mathcal{N}} \underline{\beta}_{i}\left(\Delta \omega_{i}+\Delta \lambda_{i}\right)^{2}
\end{aligned}
$$

in a neighborhood of the equilibrium. By condition (iii), we can construct $V_{i}$ in (36) using the approach in [12], such that $\dot{V}=\dot{V}_{0}+\sum_{i \in \mathcal{G}} \dot{V}_{i}$

$$
\begin{aligned}
\leq & -\Delta \omega^{T} D \Delta \omega-\sum_{i \in \mathcal{L}} \underline{\beta}_{i}\left(\Delta x_{i}\right)^{2} \\
& +\sum_{i \in \mathcal{G}}\left\{-\left(\underline{\beta}_{j}-\beta_{j}\right)\left(\Delta x_{i}\right)^{2}+\Delta x_{i} \Delta p_{i}^{M}-\alpha_{i}\left(\Delta p_{i}^{M}\right)^{2}\right\} \\
& -\sum_{i \in \mathcal{G}} \gamma_{i}\left(\Delta a_{i}+\eta_{i} \Delta p_{i}^{M}\right)^{2}
\end{aligned}
$$

where $\Delta x_{i}:=\Delta \omega_{i}+\Delta \lambda_{i}$. Constants $\alpha, \beta, \gamma, \eta$ make $\dot{V} \leq 0$, and $\dot{V}=0$ if and only if $\Delta \omega=\Delta \lambda=0, \Delta p_{\mathcal{G}}^{M}=\Delta a_{\mathcal{G}}=0$. Hence $V$ is a Lyapunov function. The rest of the proof uses the same approach as in the proofs of [10, Lemma 5, Theorem $1]$.

Theorem 2 states the stability result for closed-loop equilibria which satisfy $\tilde{\theta}^{*}=\tilde{\phi}^{*} \in \tilde{\Theta}$. In practice, the initial values of $\theta$ and $\phi$ can be configured such that their trajectories are close enough to or within $\tilde{\Theta}$, if the disturbance is sufficiently small. Moreover, the conditions in Theorem 2 are satisfied in practice in the following manner: (i) the controllable loads have large enough operating regions (control capacities) such that they do not hit their bounds at equilibrium; (ii) the load control gains are large enough and (iii) the generator control gains are small enough, around the equilibrium. We remark that the condition in Theorem 2 is sufficient for stability and is conservative. It is our future work to find less conservative stability conditions.

\section{Simulation}

We simulate the network in Fig. 1 with Power System Toolbox [14]. In the simulations, we use a more realistic

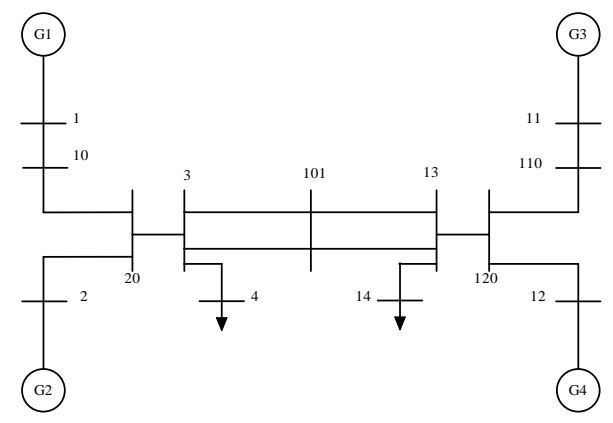

Fig. 1. The network we simulate. This figure is from [14].

model than our model in Sections II-V, including subtransient generator model, exciters and power system stabilizers, lossy lines, voltage and reactive power dynamics, et cetera. Network parameters are slightly modified from [14].

We use generation cost functions $G_{i}\left(p_{i}^{c}\right)=a_{i}\left(p_{i}^{c}\right)^{2}$, and user utility functions $U_{i}\left(d_{i}\right)=-a_{i}\left(d_{i}-\bar{d}_{i}\right)^{2}+a_{i}\left(\underline{d}_{i}-\bar{d}_{i}\right)^{2}$, for constant $a_{i}>0$. Then the control functions are $p_{i}^{c}(x)=$ $-x /\left(2 a_{i}\right)$ for $x \in\left[-2 a_{i} \bar{p}_{i},-2 a_{i} p_{i}\right]$ for generators, and $d_{i}(x)=x /\left(2 a_{i}\right)+\bar{d}_{i}$ for $x \in\left[2 a_{i}\left(\underline{d}_{i}-\bar{d}_{i}\right), 0\right]$ for controllable loads. We set $\left[\underline{p}_{i}, \bar{p}_{i}\right]$ and $\left[\underline{d}_{i}, \bar{d}_{i}\right]$ such that the generators and controllable loads do not hit their capacity limits. Let the load on bus 14 make a step increase at $t=2 \mathrm{~s}$. In different cases below, we implement different control strategies. Generators and loads are controlled once per second in all the cases. 
We first compare the proposed OLC scheme with traditional automatic generation control (AGC) [1], [3]. For simplicity, we regard the network as a single area and ignore interarea power exchange constraints, in which case AGC becomes a centralized integral control. For both OLC and AGC, all the bus frequencies are restored to $60 \mathrm{~Hz}$. Fig. 2 shows the frequency of bus 12 under AGC and OLC. The control gains of AGC and OLC are tuned such that the frequency shows best transient within each case. We see that OLC improves

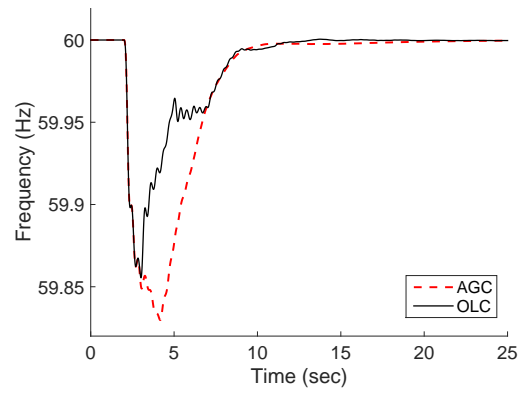

Fig. 2. Frequency of bus 12 under AGC and OLC. Control gains are tuned for best transient frequency within each case. All the four generators and two loads are controlled in both cases.

transient frequency compared to AGC.

We then look at the impact of load participation in OLC. Fig. 3 shows the frequency of bus 12 under two cases: (1) all the four generators are controlled, and (2) generators G1 and G3 and two loads on buses 4 and 14 are controlled. In both cases, OLC is used with constants $a_{i}$ tuned such that the aggregate control gain over the network is the same. We see

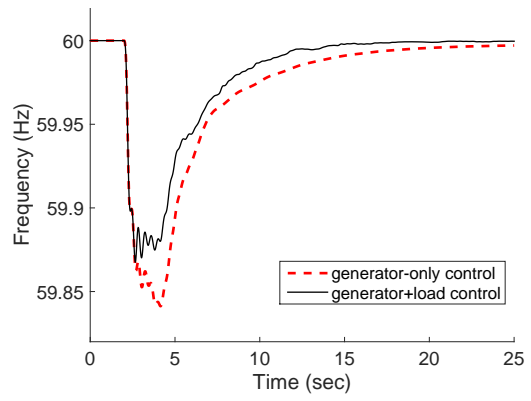

Fig. 3. Frequency of bus 12 under cases (1) only generators are controlled and (2) both generators and loads are controlled. Both cases use OLC, and have the same aggregate control gain.

that load participation in OLC improves transient frequency.

\section{CONCLUSION AND FUtURE WORK}

We have designed a distributed secondary frequency control scheme which operates jointly on generators and controllable loads, based on local sensing and computation and neighborhood communication. Equilibrium and stability analysis with a relatively realistic power network model has proved that the proposed control is able to stabilize the system, restore frequency and inter-area power exchanges, and minimize total generation cost minus user utility at equilibrium, after an unexpected change in power supply or demand. Simulations with a more realistic model has confirmed the effectiveness of the proposed scheme.
In the future, we will understand more about the performance of the proposed scheme, e.g., less conservative stability conditions, attraction region, robustness to measurement and actuation delays, modeling inaccuracies and parameter and input uncertainties. Based on these understandings, we may improve the design of the current scheme.

\section{ACKNOWLEDGMENT}

This work was supported by ARPA-E grant DEAR0000226 and Los Alamos National Lab through an DoE grant.

\section{REFERENCES}

[1] A. R. Bergen and V. Vittal, Power Systems Analysis, 2nd ed. Prentice Hall, 2000

[2] M. Andreasson, D. V. Dimarogonas, K. H. Johansson, and H. Sandberg, "Distributed vs. centralized power systems frequency control," in Proc. of European Control Conference, Zürich, Switzerland, 2013, pp. 3524 3529.

[3] N. Li, L. Chen, C. Zhao, and S. H. Low, "Connecting automatic generation control and economic dispatch from an optimization view," in Proc. of American Control Conference, Portland, OR, USA, 2014, pp. 735-740.

[4] M. Bürger, C. De Persis, and S. Trip, "An internal model approach to (optimal) frequency regulation in power grids," arXiv preprint arXiv:1403.7019, 2014.

[5] F. Dörfler, J. Simpson-Porco, and F. Bullo, "Breaking the hierarchy: Distributed control \& economic optimality in microgrids," arXiv preprint arXiv:1401.1767, 2014.

[6] F. C. Schweppe, R. D. Tabors, J. L. Kirtley, H. R. Outhred, F. H. Pickel, and A. J. Cox, "Homeostatic utility control," IEEE Trans. Power App. Syst., vol. PAS-99, no. 3, pp. 1151-1163, 1980.

[7] J. A. Short, D. G. Infield, and L. L. Freris, "Stabilization of grid frequency through dynamic demand control," IEEE Trans. Power Syst., vol. 22, no. 3, pp. 1284-1293, 2007.

[8] D. Hammerstrom et al., "Pacific Northwest GridWise testbed demonstration projects, part II: Grid Friendly Appliance project," Pacific Northwest Nat. Lab., Tech. Rep. PNNL-17079, October 2007.

[9] D. S. Callaway and I. A. Hiskens, "Achieving controllability of electric loads," Proceedings of the IEEE, vol. 99, no. 1, pp. 184-199, 2011.

[10] C. Zhao, U. Topcu, N. Li, and S. Low, "Design and stability of loadside primary frequency control in power systems," IEEE Trans. Autom. Control, vol. 59, no. 5, pp. 1177-1189, 2014.

[11] E. Mallada, C. Zhao, and S. H. Low, "Optimal load-side control for frequency regulation in smart grids," in Proc. of Allerton Conf. on Comm., Control, and Comp., Monticello, IL, USA, 2014, pp. 731-738.

[12] C. Zhao and S. H. Low, "Optimal decentralized primary frequency control in power networks," in Proc. of Conference on Decision and Control, Los Angeles, CA, USA, 2014, pp. 2467-2473.

[13] X. Zhang and A. Papachristodoulou, "Redesigning generation control in power systems: Methodology, stability and delay robustness," in Proc. of Conference on Decision and Control, Los Angeles, CA, USA, 2014.

[14] J. Chow and G. Rogers, "Power system toolbox," Cherry Tree Scientific Software, 2000.

[15] S. P. Boyd and L. Vandenberghe, Convex optimization. Cambridge University Press, 2004.

[16] A. Araposthatis, S. Sastry, and P. Varaiya, "Analysis of power-flow equation," International Journal of Electrical Power \& Energy Systems, vol. 3, no. 3, pp. 115-126, 1981. 